\title{
Molecular identification and phylogenetic analysis of human Trichostrongylus species from an endemic area of Iran
}

\author{
Meysam Sharifdini ${ }^{\mathrm{a}}$, Sedigheh Derakhshani ${ }^{\mathrm{b}, \mathrm{c}}$, Safar Ali Alizadeh ${ }^{\mathrm{c}}$, Laleh Ghanbarzadeh ${ }^{\mathrm{b}, \mathrm{c}}$, \\ Hamed Mirjalali ${ }^{\mathrm{d}}$, Iraj Mobedi ${ }^{\mathrm{e}}$, Mehrzad Saraei ${ }^{\mathrm{b}, \mathrm{c}, *}$ \\ a Department of Medical Parasitology and Mycology, School of Medicine, Guilan University of Medical Sciences, Rasht, Iran \\ b Department of Parasitology and Mycology, School of Medicine, Qazvin University of Medical Sciences, Qazvin, Iran \\ c Cellular and Molecular Research Center, Qazvin University of Medical Sciences, Qazvin, Iran \\ d Foodborne and Waterborne Diseases Research Center, Research Institute for Gastroenterology and Liver Diseases, Shahid Beheshti University of Medical Sciences, Tehran, \\ Iran \\ e Department of Medical Parasitology and Mycology, School of Public Health, Tehran University of Medical Sciences, Tehran, Iran
}

\section{A R T I C L E I N F O}

\section{Keywords:}

Trichostrongylus

Human

PCR

ITS2-rDNA region

Phylogenetic analysis

Guilan

Iran

\begin{abstract}
A B S T R A C T
Human infections with Trichostrongylus species have been reported in most parts of Iran. The aim of this study was the identification, molecular characterization and phylogenetic analysis of human Trichostrongylus species based on ITS2 region of ribosomal DNA from Guilan Province, northern Iran. Stool samples were collected from rural inhabitants and examined by formalin-ether concentration and agar plate culture techniques. After anthelmintic treatment, male adult worms were collected from five infected cases. Genomic DNA was extracted from one male worm of each species in every treated individual and one filariform larva isolated from each case. PCR amplification of ITS2-rDNA region was performed and the products were sequenced. Among 1508 individuals, 46 (3.05\%) were found infected with Trichostrongylus species using parasitological methods. Male worms of $T$. colubriformis, $T$. vitrinus and $T$. longispicularis were expelled from five patients after treatment. Out of 41 filariform larvae, 40 were T. colubriformis, and the other one was T. axei. Phylogenetic analysis showed that each species was placed together with reference sequences submitted to GenBank database. Intra-species similarity for all species obtained in the current study was $100 \%$. T. colubriformis was found to be probably the most common species in this region of Iran. For the first time, the authors of the present study report the occurrence of natural human infection by $T$. longispicularis in the world. Therefore, the number of Trichostrongylus species infecting human in Iran now increased to ten.
\end{abstract}

\section{Introduction}

Trichostrongylus nematodes are primarily parasites of herbivores with a worldwide distribution. Trichostrongylosis has been reported from human in many countries, including Italy (Buonfrate et al., 2017; Cancrini et al., 1982), Iraq (Muller, 2002), France (Lattes et al., 2011), New Zealand (Wall et al., 2011), Japan (Ghadirian and Arfaa, 1975), Laos (Sato et al., 2011; Watthanakulpanich et al., 2013), Thailand (Phosuk et al., 2013), Australia (Boreham et al., 1995) and also with highest prevalence in Iran (Ghadirian and Arfaa, 1975), Egypt (Kuntz et al., 1956), and South Korea (Muller, 2002). Studies from the past to present on the epidemiology of Trichostrongyliasis in Iran have revealed several species of Trichostrongylus from different hosts including wild herbivores (Eslami et al., 2000; Eslami et al., 1979; Eslami and Nikbin, 1980) domestic ruminants (Anvari-Tafti et al., 2013; Ghadirian and Arfaa, 1975) and human (Ashrafi et al., 2015; Ghadirian and Arfaa, 1975; Gholami et al., 2015). Of eleven species infecting the human, nine species were previously reported in human in Iran among those; $T$. orientalis and T. colubriformis were detected to be more frequent (Ghadirian, 1977; Ghadirian and Arfaa, 1975; Ghadirian et al., 1974; Muller, 2002). With the exception of T. orientalis, the other species are zoonotic and transmitted to human through close-contact with herbivores (Ghadirian and Arfaa, 1975).

Conventional method based on the morphological characters of male worms is a relatively reliable technique to differentiate Trichostrongylus spp., whereas it cannot be helpful for females, larvae and eggs of the parasites (Ghadirian and Arfaa, 1975; Phosuk et al., 2013).

Human infections mainly occur by ingesting the vegetables containing filariform larvae or occasionally through the skin penetration by

\footnotetext{
* Corresponding author at: Department of Parasitology and Mycology, School of Medicine, Qazvin University of Medical Sciences, Qazvin, Iran, P. O. Box: $34197-59811$.

E-mail address: msaraei@qums.ac.ir (M. Saraei).
} 